\title{
Tumor-induced osteomalacia caused by head and neck tumors: report of an unusual case and literature review
}

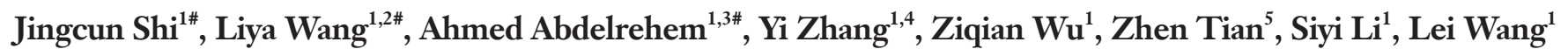 \\ ${ }^{1}$ Department of Oral and Maxillofacial-Head \& Neck Oncology, Shanghai Ninth People's Hospital, College of Stomatology, Shanghai Jiao Tong \\ University School of Medicine, Shanghai, China; ${ }^{2}$ Department of Stomatology, the First Affiliated Hospital of Soochow University, Suzhou, China; \\ ${ }^{3}$ Department of Craniomaxillofacial and Plastic Surgery, Faculty of Dentistry, Alexandria University, Alexandria, Egypt; ${ }^{4}$ Department of Stomatology, \\ Zhongshan Hospital of Xiamen University, Xiamen, China; ${ }^{5}$ Department of Oral Pathology, Shanghai Ninth People's Hospital, Shanghai Jiao Tong \\ University School of Medicine, Shanghai, China \\ \#These authors contributed equally to this work. \\ Correspondence to: Lei Wang, MD, PhD; Siyi Li, MD, PhD. Department of Oral \& Maxillofacial-Head \& Neck Oncology, Ninth People's Hospital, Shanghai \\ Jiao Tong University School of Medicine, 639 Zhizaoju Road, Shanghai 200011, China. Email: wanglei@sh9hospital.org.cn; dr.lisiyi@hotmail.com.
}

Background: Tumor-induced osteomalacia is a relatively rare paraneoplastic syndrome. Fewer than 200 cases of tumor-induced osteomalacia following head and neck tumors have been reported worldwide. Herein, we report a case of tumor-related osteomalacia caused by a maxillofacial tumor, and review the literature in order to enhance clinicians' understanding of tumor-induced osteomalacia and propose diagnostic strategies.

Case Description: In this case, the patient presented with bone pain first in the left thigh region and later involved the ribs and ankle, which had been mistakenly treated as being ankylosing spondylitis without any obvious effect. The symptoms worsened later and the patient was unable to walk. Laboratory tests showed that serum phosphorus decreased, while alkaline phosphatase and parathyroid hormone increased. Moreover, 25 hydroxyvitamin D and serum calcium were normal. The patient was diagnosed as osteomalacia in another hospital, and the subsequent positron emission tomography computed tomography examination suggested focal bony destruction of the left maxilla with an increased radioactive uptake. After hospital admission, the patient underwent "resection of the left maxillary mass + radical resection of the left maxillary sinus". The postoperative pathology indicated phosphaturic mesenchymal tumor; mixed epithelial and connective tissue type. On the 4th postoperative day, the serum phosphorus level of the patient returned to normal, the sensation of bone pain and joint stiffness gradually improved, and the patient became more flexible than before surgery.

Conclusions: Surgeons can learn the followings from this case report: (I) if osteomalacia is found, we should carefully distinguish whether it is genetic or secondary, carefully inquire whether there is a family history and special medical history, and carefully consider physical examination and imaging evaluation to localize the inducing tumor as soon as possible; (II) tumor-induced osteomalacia is mostly benign or lowgrade malignancy, with a rare incidence of high-grade malignancy. However, due to its local invasive nature, an extensive resection is often performed around the tumor to reduce recurrence. In a word, this case report is worthy that it addresses the common misleading presentation of such cases and subsequent mistreatment as osteoporosis that inversely affects prognosis.

Keywords: Tumor-induced osteomalacia (TIO); oncogenic osteomalacia; phosphaturic mesenchymal tumor (PMT); head and neck; case report

Received: 07 March 2021; Accepted: 30 April 2021; Published: 10 June 2021.

doi: $10.21037 /$ fomm-21-23

View this article at: http://dx.doi.org/10.21037/fomm-21-23 


\section{Introduction}

Clinically, hypophosphatemia can be diagnosed when the serum phosphorus level is less than $0.8 \mathrm{mmol} / \mathrm{L}$. Considering therapeutic aspects, when the serum phosphorus level is reduced to $0.32 \mathrm{mmol} / \mathrm{L}$, then phosphorus supplementation should be given through intravenous routes. Hypophosphatemia is not uncommon and can occur in all individuals with no age or gender limitations, with various range of clinical presentations ranging from asymptomatic to severe manifestations. Hypophosphoric osteomalacia is characterized by low blood phosphorus and deficiency of active vitamin $\mathrm{D}$, therefore, clinically resulting in muscle weakness, muscle paralysis, bone pain, rickets, and pathological fractures. Other reported reasons for low blood phosphorus include: (I) reduced phosphorus intake or malabsorption; (II) excessive renal phosphorus excretion; (III) phosphorus transfer towards the cells. Clinically, chronic use of certain drugs (such as adefovir dipivoxil) or the presence of certain tumor entities may also lead to secondary osteomalacia.

Tumor-associated osteomalacia was first reported by McCance in 1947 (1), however, without indicating a definite relationship between tumors and osteomalacia. In 1959, Prader $e t a l$. concluded that a defined tumor entity was the underlying cause of osteomalacia with low phosphorus levels, and the disease was relieved after tumor resection (2). Folpe et al. found through a retrospective study, that $88 \%$ of the pathological manifestations of tumor-induced osteomalacia (TIO) were due to phosphaturic mesenchymal tumor (PMT) (3). The first case of TIO in China was reported in 1981, caused by a mesenchymal tumor located in the groin. After tumor resection, the blood phosphorus level quickly returned to normal and the symptoms were relieved within few months. To date, less than 200 cases of osteomalacia caused by maxillofacial tumors have been reported in literature (4). There are two main reasons for the low number of reported cases: first, the prevalence of TIO is low and less cases occur in the maxillofacial region; second, clinicians are poorly aware of the relationship between osteomalacia and mesenchymal tumors.

TIO-responsible tumor is often involved in secretion of several phosphorus-promoting factors: fibroblast growth factor-23 (FGF-23), matrix extracellular phosphoglycoprotein (MEPE) and secreted frizzled related protein 4 (sFRP4) substances, such as promoting renal tubular excretion of phosphate, and inhibiting the production of 1,25 vitamin $D$. This leads to hypophosphatemia $(3,5,6)$, which can result in an insufficient bone mineralization and osteomalacia, clinically manifested as generalized bone pain, muscle weakness, skeletal deformity or fractures, and movement disorders, seriously affecting the quality of life $(7,8)$. Laboratory tests often showed an increased urinary phosphorus, decreased blood phosphorus, increased serum alkaline phosphatase (ALP), and increased FGF-23. Bone X-ray films showed decreased bone density, rough trabeculae, marginal bone destruction, bone cortical discontinuity or disappearance, and sometimes pseudo fractures (9). Tumors that cause hypophosphatemia are usually slow growing, concealed, and having no definite characteristic manifestations. When the tumor is very small, it is difficult to diagnose, and this is often mistakenly treated as an osteoporosis, which delays the disease. In this study, a case of osteomalacia caused by a tumor occurring in the jaw bones was reported. Local and English language literatures were reviewed to summarize its characteristics, so as to improve clinician's understanding of TIO and different perspectives of diagnosis and treatment. We present the following case in accordance with the CARE reporting checklist (available at https://fomm. amegroups.com/article/view/10.21037/fomm-21-23/rc).

\section{Case presentation}

A 25-year-old female patient, was admitted to the hospital due to osteomalacia for 3 years. Clinically, the patient presented with multiple bone pain, such as ribs and ankles, accompanied with reduced mobility, without an obvious inducement. Radiological assessment revealed left maxillary lesion for three months. On October 2018, the patient underwent treatment for ankylosing spondylitis, however, the symptoms worsened and the patient was then unable to walk. The serum phosphorus was 0.52 and $0.63 \mathrm{mmol} / \mathrm{L}$ on April, 2019. Computed tomography (CT) examination revealed lesion involvement in bilateral sacroiliac joints, symphysis pubis and bilateral ribs, with no obvious abnormalities using bone marrow puncture. Three weeks later, positron emission tomography computed tomography (PET-CT) examination showed an active bone metabolism locally in the mandible, bilateral junctions of multiple ribs, both sides of multiple thoracic vertebrae, bilateral middle humerus, bilateral hip joints, pubic symphysis, and bilateral ankle joints. Scintigraphy scanning was performed on May 20, 2019, and no obvious abnormality was found. Laboratory investigations were as follow: parathyroid hormone (PTH) was $121.4 \mathrm{pg} / \mathrm{mL}(12-88 \mathrm{pg} / \mathrm{mL})$, total 25 vitamin 


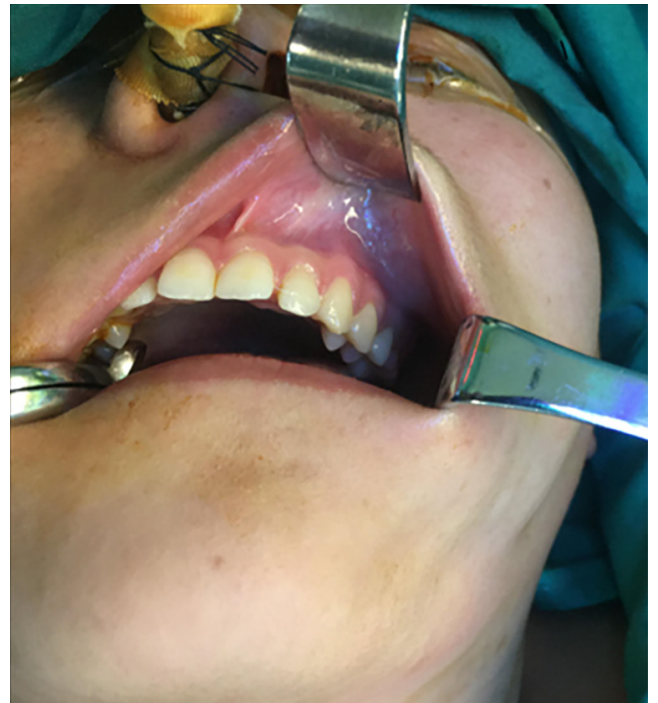

Figure 1 There was no obvious apophysis or defect in the left maxillary area, no obvious loosening of the left upper and posterior teeth, no redness and swelling of the gingiva, and no obvious abnormality of the oral mucosa. The absence of tumor on physical examination may be one of the reasons for the delay in treatment.

D was $20.76 \mathrm{ng} / \mathrm{mL}(>20 \mathrm{ng} / \mathrm{mL})$, ALP was $201 \mathrm{U} / \mathrm{L}$ (15-116 U/L), serum phosphorus was $0.46 \mathrm{mmol} / \mathrm{L}$ (0.8-1.6 mmol/L), and serum calcium was $2.21 \mathrm{mmol} / \mathrm{L}$ $(2.08-2.65 \mathrm{mmol} / \mathrm{L})$. From the previous clinical and laboratory measures, the patient was then diagnosed as osteomalacia in another hospital. Following symptomatic treatment with phosphorus solution, the symptoms improved significantly. During PET-CT examination in another hospital in May 2020, focal bone destruction involving the left maxilla was found, and the patient was admitted to our hospital as "osteomalacia with left maxillary lesion" in the outpatient department. The patient was in good health, reporting negative family genetic history, and having no history of exposure to toxicant or application of special drugs.

Physical examination: the facial form and shape were basically symmetrical, with no palpation of obvious apophysis or defect in the left maxillary area. Additionally, no obvious loosening of the left upper and posterior teeth, no knocking pain, no gingival swelling, no obvious abnormality of the oral mucosa, and no obvious neck nodal swellings (Figure 1). Imaging examination: PET-CT showed focal destruction of the left maxilla with a diameter of about $1.8 \mathrm{~cm}$. Hyperosteogeny of cervical, thoracic and lumbar vertebral bodies was also a remarkable finding. Contrast- enhanced CT showed a space-occupying lesion in the left maxilla (Figure 2).

After admission, "resection of the left maxillary mass + radical resection of the left maxillary sinus" was performed under general anesthesia (Figure 3). Postoperative pathological diagnosis revealed a short spindle cell lesion involving the left maxilla, accompanied with hemorrhage and osteogenesis. Combined with the medical history, it was considered to be PMT; mixed epithelial and connective tissue type (PMTMECT) (Figure 4). All calcium, phosphorus and vitamin $\mathrm{D}$ preparations were stopped immediately after surgery. The patient's blood inorganic phosphorus level returned to normal 4 days after operation. One week after the operation, the level of inorganic phosphorus in blood was normal, the wound healing in the operative area was good, the feeling of bone pain and joint stiffness were significantly improved, and the self-movement was more flexible than before. The patient was followed up for 5 months, during which the blood phosphorus level continued to be normal, with no obvious abnormality observed in the operative area. Systemic symptoms were significantly improved and the patient could move freely. Blood biochemical and bone transformation indices were shown in Table 1.

Bone mineral density (BMD) examination with dualenergy X-ray absorptiometry was performed revealing BMD values of lumbar spines and femoral neck were 0.753 and $0.646 \mathrm{~g} / \mathrm{cm}^{2} 16$ months before surgery. After 1.5 months of symptomatic treatment with neutral phosphorus solution in another hospital, BMD values of lumbar spines and femoral neck were both increased with clinical significance, which indicated that symptomatic treatment was effective. BMD and $T$ value were not significantly changed after 1 month of withdrawal of all calcium, phosphorus and vitamin D preparations after surgery, suggesting that BMD was at a relatively stable level after tumor resection, and the disease was under control without further progression. However, osteoporotic bone mass remained at the spine ( $\mathrm{t}$-score: -2.6 ), and osteopenia remained at the femoral neck ( $t$-score: -1.9 to -2.0$)$. The results of BMD examination before and after surgery were shown in Table 2 .

All procedures performed in studies involving human participants were in accordance with the Declaration of Helsinki (as revised in 2013). Approved by the Medical Ethics Committee of Shanghai Ninth People's Hospital, Shanghai Jiao Tong University School of Medicine (SH9H2018-T95-2). The patient's permission for publication was obtained. 

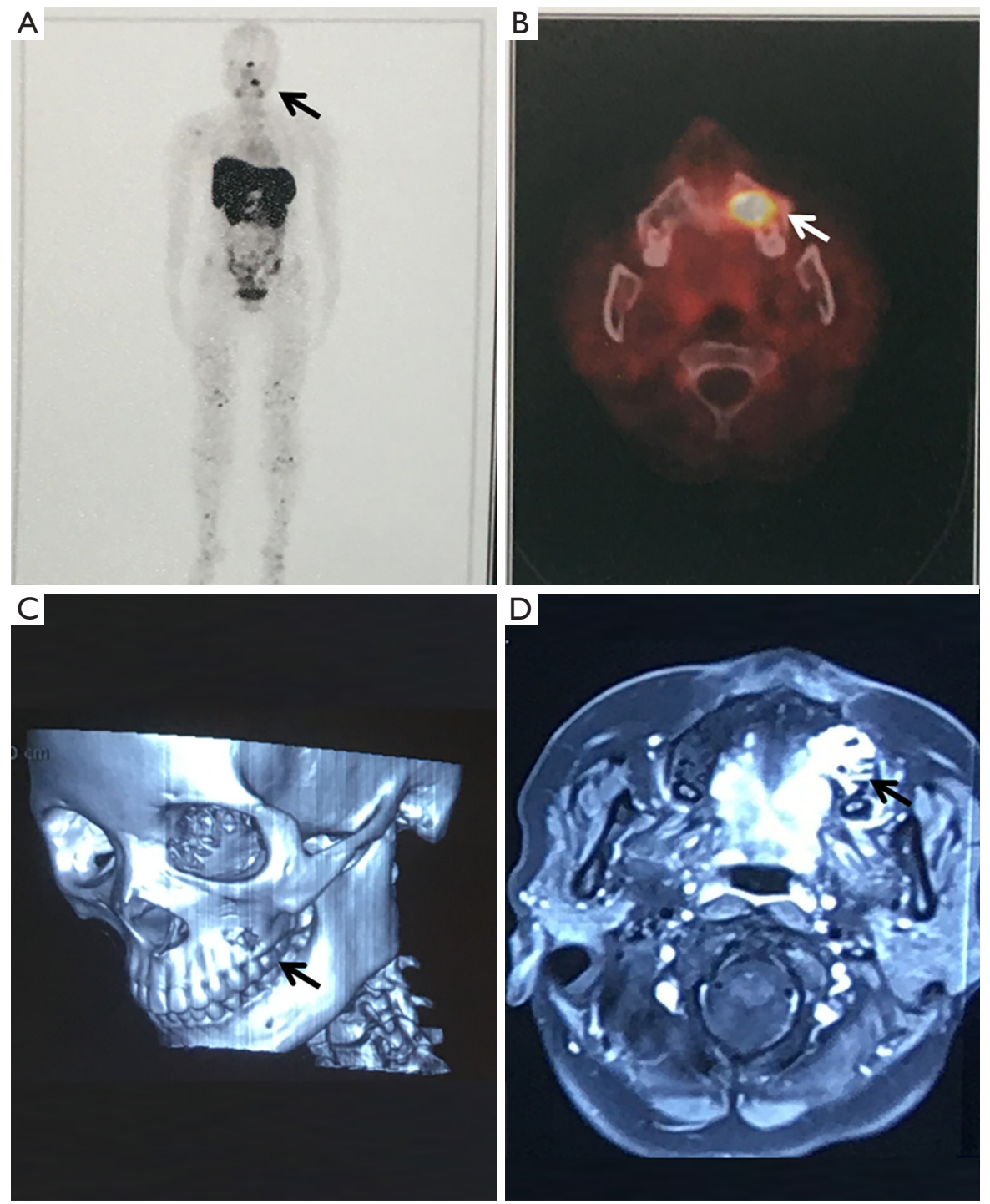

Figure 2 Imaging manifestations of TIO-responsible tumor. (A,B) PET-CT indicated focal bone destruction of the left maxilla with an increased radiation uptake; (C,D) enhanced CT indicated a space-occupying lesion in the left maxilla (the arrow indicates the site of the lesion). TIO, tumor-induced osteomalacia; PET, positron emission tomography; CT, computed tomography.

\section{Discussion}

\section{Incidence/epidemiology}

TIO occurring mainly in adults and accidentally in children. TIO patients aged 30 years old and above accounted for more than two-thirds of the total cases, however without any obvious difference in the incidence ratio. Most of the cases are single lesion, however it has been reported that few patients (2\%) have multiple lesions, distant metastasis cases included (6). TIO can occur in either soft or bony tissues (10), with no significant differences in both tissues. Most of the tumors occur in the extremities, craniofacial region $(6,11)$. Within the head and neck region, the most common parts are the paranasal sinuses, upper and lower jaws, cranial fossa, gums, tongue, and buccal vestibular sulcus. Moreover, an involvement of the skull bones, parotid gland, neck, infratemporal fossa, mastoid process, thyroid gland, and vertebral bodies has also been reported (4). The average tumor size reported in the literature was $1-14 \mathrm{~cm}(3,12)$. The current case reported here demonstrated a tumor diameter of about $1.8 \mathrm{~cm}$ involving the maxilla. Physical examination did not reveal the mass, which was consistent 

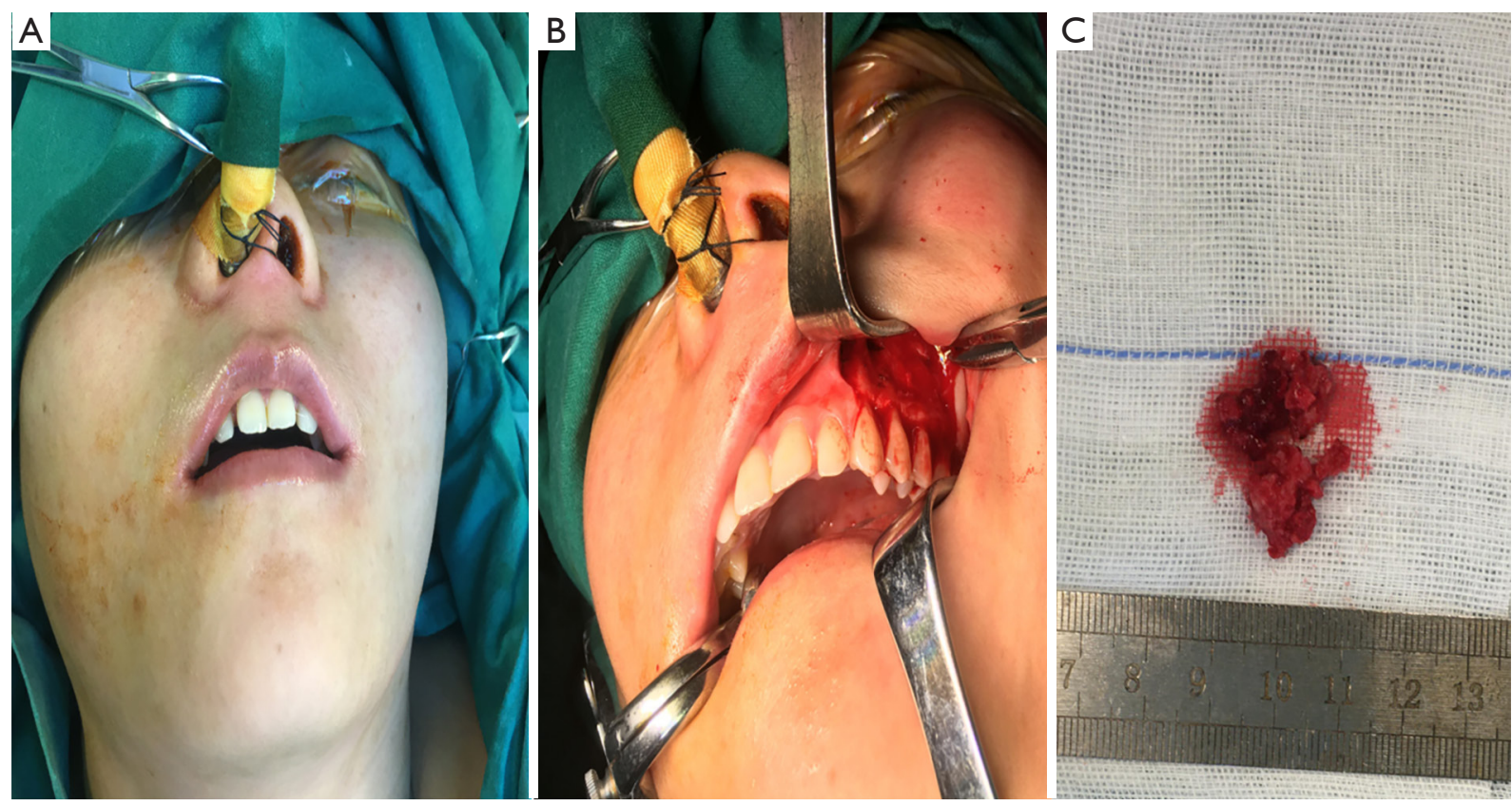

Figure 3 Preoperative and postoperative photos of the patient and the resected mass. (A) Preoperative facial profile of the patient showing symmetry. (B) Exposure of the lesion through upper sulcus incision and total excision. (C) The mass was removed surgically. Grossly, the mass was composed of broken tissue, with yellowish cyst walls and hard tissue visible.

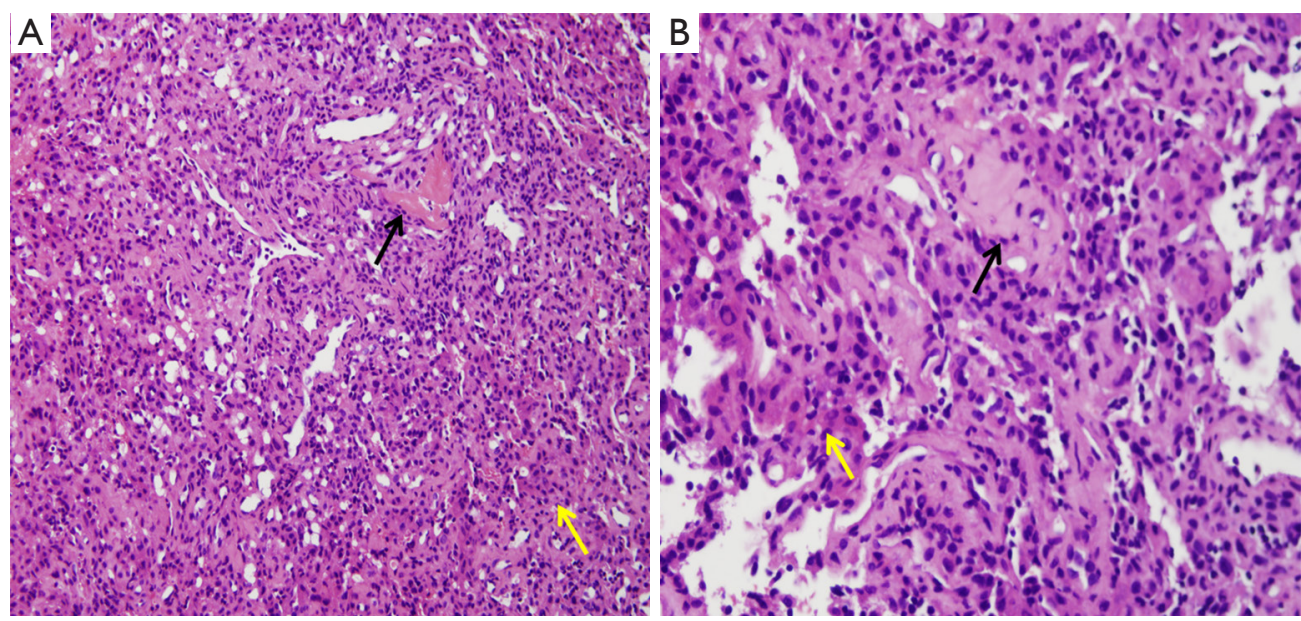

Figure 4 Left maxillary lesion hematoxylin and eosin-stained. (A) The tumor tissue was rich in blood vessels, focal "staghorn" branching pattern of blood vessels were observed, focal osteoid matrix was commonly appreciated ( $\times 200)$. (B) The mesenchymal component was composed of spindle cells, the neoplastic epithelial cells had eosinophilic $(\times 400)$. The yellow arrows indicate eosinophilic epithelial cells; the black arrows indicate osteogenesis and calcification. 
Table 1 Patient laboratory results

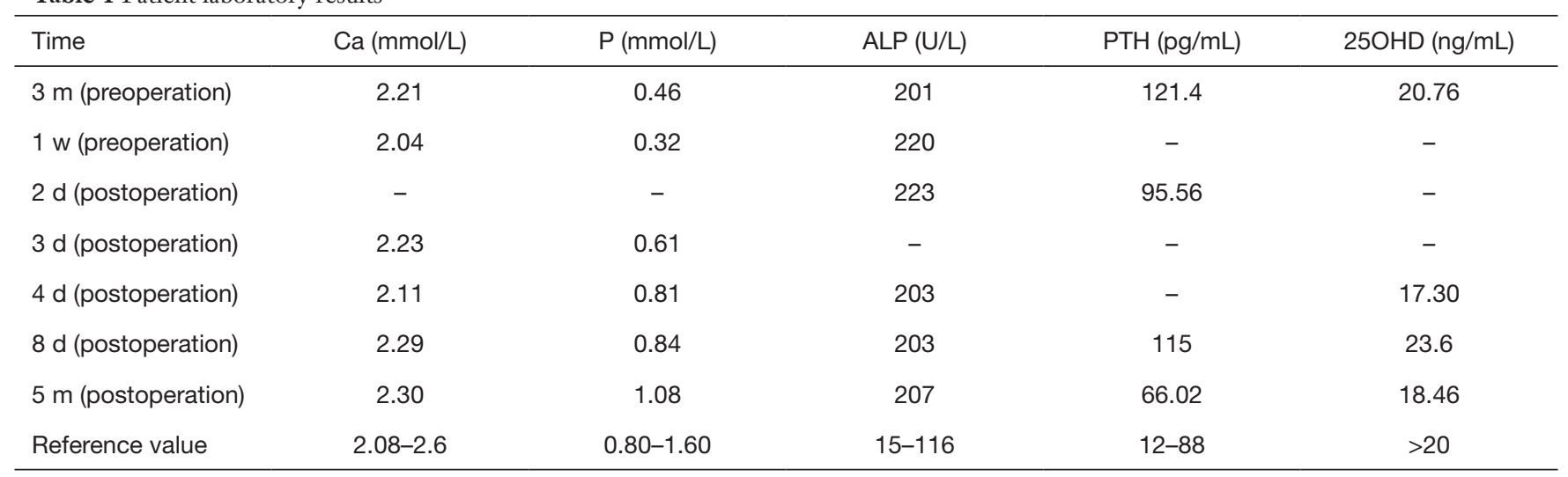

Ca, serum calcium; P, serum phosphorus; ALP, alkaline phosphatase; PTH, parathyroid hormone; 25OHD, 25-hydroxyvitamin D.

Table 2 Bone mineral density examination before surgery and 1 month after surgery

\begin{tabular}{|c|c|c|c|c|}
\hline Time & \multicolumn{2}{|c|}{$\mathrm{BMD}\left(\mathrm{g} / \mathrm{cm}^{2}\right)$} & \multicolumn{2}{|c|}{$\mathrm{T}$ value score } \\
\hline 16 m (preoperation) & 0.753 & 0.646 & -3.0 & -1.3 \\
\hline $2.5 \mathrm{~m}$ (preoperation) & $0.805^{\star}$ & $0.700^{\star}$ & -2.6 & -1.9 \\
\hline 1 m (postoperation) & 0.802 & 0.686 & -2.6 & -2.0 \\
\hline
\end{tabular}

*, indicates a significant change based on a 95\% confidence interval. The diagnostic criteria for osteoporosis (T-value score) are normal bone mass: above -1 ; decreased bone mass: -1 to -2.5 ; osteoporosis: below -2.5 ; severe osteoporosis: less than -2.5 with one or more fractures. BDM, bone density.

with the characteristics of small size and hidden lesions of the TIO-responsible tumor.

\section{Pathogenesis}

The pathogenesis of TIO is mainly related to tumors secreting FGF-23, which inhibit the proximal renal tubular sodium phosphate transporters and 1- $\alpha$-hydroxylase, leading to an increase in the rate of phosphate excretion and vitamin D treatment resistance (13-15). Under normal circumstances, the blood phosphorus levels would enhance renal $1,25(\mathrm{OH})_{2} \mathrm{D}_{3}$ hydroxylase activity, therefore maintaining the blood level of $1,25(\mathrm{OH})_{2} \mathrm{D}_{3}$, hence increasing the gastrointestinal absorption of phosphorus, which improves the low phosphorus concentration. In case of TIO, FGF-23 inhibits 1 - $\alpha$-hydroxylase activation of vitamin $\mathrm{D}$, with a decreased level of $1,25(\mathrm{OH})_{2} \mathrm{D}_{3}$ in the blood as a result, aggravating the hypophosphatemia. In addition, other studies have found that FGF-23 can directly inhibit the differentiation of osteoblasts and calcification of bone stroma (16). Studies have confirmed that TIO is rich in the tumor tissues with FGF-23 mRNA expression. Furthermore, in $70 \%$ of cases, FGF-23 antigen expression in tumor tissues is positive, and FGF-23 usually comes from proliferating cells (3). The preoperative serum FGF-23 is significantly higher than normal levels. The postoperative serum phosphorus returns earlier to normal, however, otherwise the serum FGF-23 is also maintained at a higher level. MEPE mRNA is highly expressed in the tumor tissues of TIO patients, and is also positively correlated with FGF23 mRNA, suggesting that MEPE mRNA plays a role in the pathogenesis. SFRP4 is expressed in tumor tissues of only part of TIO patients. SFRP4 is also expressed in other bone tissues and mesenchymal tissue derived tumors, so its role in the pathogenesis needs further investigation (17).

\section{Clinical feature}

The typical clinical manifestations of TIO for bone pain are progressive development, pain in limbs and weight-bearing joints at first, gradual progression involving the spine and ribs, often accompanied with skeletal malformations 
(scoliosis and pelvic deformation), reduced $\mathrm{BMD}$, may be associated with pathologic fractures, shortened height, muscle pain, muscle atrophy, weakness of limbs, limited activity, and even difficulty to get out of bed, which seriously influence the patient's quality of life. Routine phosphorus and vitamin D supplementation have poor effects. Early in the course of disease, tumors are often misdiagnosed as ankylosing spondylitis, osteoporosis, lumbar disc herniation, etc. $(10,18)$, as often being small in size and hidden at earlier stages. Clinical symptoms and biochemical examination of blood phosphorus are usually significantly improved in a short period after tumor resection. A retrospective study found that about $50 \%$ of patients showed signs of tenderness and/or swelling at the proposed site of the tumor or swelling. Therefore, careful physical examination can help early detection of tumors in TIO patients (4). Tumors in jaw can lead to pathological loosening of teeth, fall off, sometimes slightly bulging jaw. Soft tissue tumors in the oral cavity are often associated with visible local gingival or mucosal thickening, bulge, intact mucosal surface, no erosion or ulcer, normal color, and tough texture. The case reported here started with pain in the left thigh, which later involved the ribs and ankle, and gradually worsened until the patient was unable to walk. It was misdiagnosed at first as ankylosing spondylitis. During physical examination, there was no obvious defect or bulge in the left maxilla, no obvious loosening or knocking pain in the left upper and posterior teeth, normal mucosal color, with no obvious signs that might be correlated to the small size of the mass and less severe damage of the jaw bone.

\section{Imaging manifestations}

In TIO patients, X-ray examination reveals reduced bone mass, thinning, discontinuity or disappearance of bone cortex, vague and rough texture of bone trabecula, pelvic and vertebral deformations, pseudo fractures and other imaging manifestations. When it occurs in children, this can be manifested as widening of the long shaft endings, with no clear edges. Some scholars retrospectively studied TIO patients and found that the CT imaging features of tumors occurring in bone tissues were as follows: focal osteolytic bone destruction or locally increased bone density, with an eccentric growth due to involvement of long bones with lesions, and an occasional cortical bone involvement. Most tumors involving soft tissues present as nodules of uniform density or even low density (19). Because TIO-responsible tumors are usually small in size, slowly growing and concealed in location, with an involvement of either bone or soft tissues, conventional imaging examinations such as $\mathrm{X}$-ray, CT and MRI can only examine local areas, which is easy to misdiagnose. If the tumor involves the jaw bone, the panoramic film can reveal the bone cortical interruption with an absent bone trabecula. If the lesion is closely related to teeth, it can show an irregular and ill-defined apical region.

In 1996, Reubi et al. (20) reported the expression of somatostatin receptors in various mesenchymal tissue derived tumors. Therefore, somatostatin receptor imaging is one of the commonly used imaging methods for locating TIO-responsible tumors, including ${ }^{111}$ in-octreotide scintigraphy, ${ }^{99 \mathrm{~m}} \mathrm{Tc}$-hynic-octreotide $\left({ }^{99 \mathrm{~m}} \mathrm{Tc}\right.$-hynic-TOC), and ${ }^{68} \mathrm{GA}$-DOTA-Tate-based (PET)-CT $\left({ }^{68} \mathrm{GA}-D O T A-\right.$ Tate PET/CT) $(21,22)$. Considering tumor detection and localization using different imaging methods, ${ }^{99 \mathrm{~m}} \mathrm{TC}-$ HYNIC-TOC shows a diagnostic sensitivity of $86.3 \%$, a specificity of $99.1 \%$, and an overall accuracy of $93.4 \%$ (23). Yan et al. performed ${ }^{99 \mathrm{~m}} \mathrm{Tc}-\mathrm{Hynic}-\mathrm{TOC}$ scan on 94 patients who met the clinical symptoms of osteomalacia, revealing 46 patients with high absorption, among which 6 patients showed no tumor after ultrasound, CT, or MRI examination, with a sensitivity of $94.9 \%$ [37/39]. In addition, 2 patients with TIO showed no abnormality on ${ }^{99 \mathrm{~m}}$ Tc-Hynic-TOC examination but the tumor was revealed by physical examination (6). Through a retrospective study, Zhang et al. found that ${ }^{68} \mathrm{GA}-\mathrm{DOTA}-\mathrm{TATE}$ PET/ CT imaging had a sensitivity of $100 \%$ [32/32], a specificity of $90.9 \%$ [10/11], and an overall detection rate of $97.7 \%$ [42/43] for osteomalacia tumors (24). Some studies on imaging diagnosis of TIO are shown in Table 3.

It has been reported that, compared with scintigraphy, PET-CT has more advantages in revealing small tumors and complex anatomical structures. This could be explained as follows: (I) the resolution of PET-CT is higher than scintigraphy, scintigraphy may miss some small lesions or lesions with low radioactive uptake capability; (II) scintigraphy is two-dimensional imaging modality, which might be limited by an overlap with some organs with high physiological radiation uptake rate, such as liver, spleen and kidney leading to missing the proposed lesions, while PETCT is a whole-body tomography (20); (III) TIO-responsible tumors can express somatostatin receptor SSTR1-5, of which SSTR2 is the most commonly expressed. Scintigraphy has higher affinity with SSTR2 and SSTR5 than other receptors, which may lead to missed diagnosis for some tumors. In addition, the binding force between 
Table 3 Review of published literature on TIO cases

\begin{tabular}{|c|c|c|}
\hline Ref. & Case number & Comments \\
\hline Qari H 2016, (25) & 55 & $\begin{array}{l}\text { PMTs occur in head and neck, extra-oral sites make up } 76 \% \text { and } 24 \% \text { located in intra-oral. Parana- } \\
\text { sal sinuses are the most common location (38\%) followed by the mandible (15\%). Nine of } 55 \text { cases } \\
\text { recurrence which included } 3 \text { malignant cases }\end{array}$ \\
\hline Shah R 2019, (4) & $\begin{array}{l}\text { Cohort } 1=7 ; \\
\text { Cohort } 2=163\end{array}$ & $\begin{array}{l}\text { Surgical excision is the majority management of TIOs, however, } 2 \text { of } 7 \text { cases received radiation } \\
\text { therapy and } 1 \text { case received peptide receptor radionuclide therapy in cohort } 1 \text {. In cohort } 2 \text { primary } \\
\text { radiotherapy }(n=2) \text { and surgery combined with radiotherapy }(n=2) \text { are reported }\end{array}$ \\
\hline
\end{tabular}

TIO, tumor-induced osteomalacia; PET, positron emission tomography; CT, computed tomography; PMT, phosphaturic mesenchymal tumor.

TATE and SSTR2 is stronger than that of TOC $(20,26,27)$. Scintigraphy tests may show false positives. If the radioactive material is concentrated lightly at the joint site, an inflammation should be considered. If the uptake site is consistent with the fracture line, this could be related to the fracture. Meanwhile, Scintigraphy tests may also have an increased radio-active uptake for coagulation necrotic tissue and hemangioma $(17,19)$. In one case reported with low blood phosphorus osteomalacia, scintigraphy scan revealed a soft tissue lesion and pathological findings suggesting a typical case of malignant nerve sheath meningioma, with the postoperative blood phosphorus maintained at low levels, hence proving scintigraphy scan is nonspecific (11), this is due to the working principle, as already mentioned; the scintigraphy is based mainly on radioactive tracer marker of somatostatin analogue combined with tumor cell surface receptor specificity, therefore such an imaging tool has higher sensitivity for neuroendocrine tumors, pancreatic endocrine tumor, breast cancer and other tumors. Two years after the onset of ostealgia in the current case, scintigraphy examination showed no significant abnormality. Three years later, 68GA-DOTA-TATE PET-CT examination revealed focal destruction of the left maxillary bone with an increased radioactive uptake. The lesion was missed with scintigraphy but was demonstrated by PET-CT examination, which may be due to the small size of the tumor.

\section{Laboratory indicators}

Laboratory indicators of low phosphorus osteomalacia patients have characteristic features as follows: significantly increased urinary phosphorus excretion, significantly lower blood phosphorus, normal blood calcium level, elevated ALP (hint of highly active osteoblasts and active bone remodeling), lower or normal blood $25(\mathrm{OH}) \mathrm{D}_{3}$ level, reduced $1,25(\mathrm{OH})_{2} \mathrm{D}_{3}$ level, occasionally aminoaciduria and diabetes. Blood phosphorus significantly increased after tumor resection, within one week postoperatively, based on literature reports, however, in $11.8 \%$ [2/17] of TIO patients, blood phosphorus can't return to normal levels after tumor resection, which can be explained presumably to be due to the incomplete resection of the tumor. Following tumor recurrence, blood phosphorus shows a remarkable decrease again, suggesting that blood phosphorus can be used as an indicator to monitor whether the tumor is completely resected and also to investigate for relapse $(11,17)$. Serum FGF-23 of TIO patients can return to normal within 2-6 h after tumor resection, therefore serum FGF-23 level can be regarded as an important indicator for TIO diagnosis and postoperative follow-up (28). Recently, some scholars proposed that the rapid detection of FGF-23 in tumor tissues of patients with TIO could be used to assist in the intraoperative diagnosis, adjust the surgical resection margins or determine the disease-free edge in the parts 
that are difficult to be excised (29). The decrease of serum $1,25(\mathrm{OH})_{2} \mathrm{D}_{3}$ level can stimulate the secretion of PTH and increase the serum PTH level. If such tumor entities are not found, then patients need to use the drug supplement such as neutral phosphate preparations for a long time. Long-term use of phosphorus will further stimulate the secretion of PTH, and then hyperparathyroidism will occur, resulting in increased blood calcium and further decreased blood phosphorus. When PTH is too high, parathyroid hyperplasia or parathyroid adenoma should be vigilant (11), and parathyroid surgery should be performed (30).

\section{Pathological features}

In 1972, Evans and Azzopardi (31) believed that the pathological manifestations of tumors causing osteomalacia were different from other mesenchymal tumors and might be a separate entity and phenotype. According to Weidner and Santa (32), the pathological features of tumors causing hypophosphoric osteomalacia were defined as "PMT" and were then classified into phosphaturic mesenchymal Tumor-mixed connective tissue variant (PMT-MCT), osteoblastoma, non-ossifying fibroma and ossifying fibroma, among which mixed connective tissue type is the most common. A retrospective analysis of 32 cases of mesenchymal tumors associated with osteomalacia was conducted and this concept was reintroduced. PMT occurring in bone and soft tissues has the following histological similarities: (I) low cell mass, osteogenic, cartilaginous, and myxoid matrix, separately or in combination; (II) grungy-like calcium salt deposits may appear in the tissues; (III) the tumor may contain adipose tissue; (IV) the tumor tissue is rich in blood vessels, with sinusoid, thin-walled or thick-walled malformed blood vessels; (V) it may contain diffuse or spindle bundled fibroblasts, osteoclast-like giant cells, myxoid cells, and chondroid cells; (VI) invasive growth can be seen in the tumor tissue. If necrosis, nuclear atypia or mitotic images are common, then malignancy is considered; (VII) there may be multinucleated giant cells, old hemorrhage, fibrous histiocytic reaction, aneurysmal bone cysts, microcysts, and membranous osteogenic margins in the tissues; (VIII) immunohistochemical staining showed strong positive for vimentin in all tumor cells, focal positive for smooth muscle actin (SMA) and CD34, negative for S-100, desmin and AE1/AE3.9. AB/PAS staining of myxoid matrix and perivascular mucinous changes were $A B$ positive $(3,11,32)$. In this case, the mass was removed and sent to the pathology department. Microscopically, spindle cell neoplasia with epithelial cells, were accompanied by bleeding and osteogenesis. Combined with the medical history, the patient was considered to be a PMTMECT.

\section{Differential diagnosis}

Hypophosphoric osteomalacia can be divided into hereditary hypophosphoric osteomalacia: autosomal dominant hypophosphatemic rickets (ADHR), autosomal recessive hypophosphatemic rickets (ARHR), X-linked hypophosphatemic tickets (XLH) and acquired hypophosphatemic osteomalacia (hypophosphoric osteomalacia secondary to Adefovir dipivoxil). Hereditary low phosphorus osteomalacia occurs at childhood onset and the majority of patients have a family history, namely ADHR in juvenile stage disease characterized by rickets. If this occurs after puberty onset, then it is characterized by osteomalacia, hereditary low phosphorus osteomalacia without clinical symptoms of muscle weakness (33). TIO most commonly occurs after puberty onset, genetic testing was performed to rule out hereditary hypophosphatemic osteomalacia (34). In addition, TIO also needs to be differentiated from brown tumors caused by hyperparathyroidism, because it can be clinically manifested as systemic osteoporosis, bone pain, fractures, and hypophosphatemia. However, primary hyperparathyroidism is accompanied by hypercalcemia. Pathological examination of brown tumors shows numerous multinucleated giant cells, and CD68 stained positive in the multinucleated giant cells. Following surgical removal of parathyroid adenoma, the jaw tumor disappeared (35). The presented case is an adult female with no history of adefovir dipivoxil or other special drugs intake, with no family history of similar conditions. The symptoms of bone pain and muscle weakness occurred at the time of disease onset, which is consistent with the characteristics of TIO.

\section{Treatment and prognosis}

For patients with TIO, after complete surgical resection of the tumor, blood phosphorus gradually returns to normal levels. Also, bone pain and movement disorders can also gradually recover, with good and smooth recovery of patients $(21,29,36)$. Sharkis et al. reported a case of TIO patient with complete remission of femoral head due to ischemia necrosis after tumor resection (37). In the current report, the patient underwent "left maxillary mass 
resection + left maxillary sinus radical resection" under general anesthesia. Then, calcium, phosphorus and vitamin D preparations were discontinued after surgery. After operation, serum phosphorus gradually returned to normal level until the $8^{\text {th }}$ day postoperatively. Surgical wound healing in the operative area was good, the sensation of bone pain and joint stiffness were significantly improved, and the self-movement was more flexible than before.

Most of the tumors responsible for TIO were benign or low-grade malignant lesions, with a very low proportion of malignancy, however, with an invasive growth nature in biological behavior. Moreover, the local invasive properties of TIO might be related to the overexpression of growth factor receptors including platelet derived growth factors type A and type B (PDGFRA \& PDGFRB), vascular growth factor receptor (VEGFR) and other related factors, which are involved in tumor angiogenesis and metastasis (38). Therefore, during surgical resection of the tumor, a wide resection should be performed to include a cuff of the surrounding normal tissues. Studies have reported that primary maxillofacial tumors were accompanied by hypophosphatemic osteomalacia, and blood phosphorus returned to normal levels after tumor resection, however, blood phosphorus decreased again 2 years later. Scintigraphy examination of the jaw showed no obvious abnormality, and there was an increased uptake area in the left scapula, which was consistent with TIO recurrence combined with course analysis (17).

Although complete surgical resection is the standard treatment for patients with TIO, radiofrequency ablation can also be an effective, safe and less invasive alternative for patients with TIO if the anatomical structures are complex, some anatomical sites are difficult to reach, surgery may affect surrounding important structures, or patient conditions are not suitable for surgery (39). When surgery and radiofrequency ablation are not available, external radiotherapy and peptide receptor radiotherapy are also conservative options for management of TIO patients (4) (Table 3). It has been reported that the treatment of one patient with somatostatin analogues can antagonize the loss of phosphate in renal tubules, opening up a new way for the treatment of patients with TIO, but some scholars have questioned this, as no definite efficacy has been observed in other TIO patients (22). A phase I clinical trial showed that anti-FGF-23 antibody effectively increased serum phosphorus levels in X-linked rickets (40), and it is believed to be promising in the treatment of TIO as well.

\section{Conclusions}

Although there are clear and distinguished diagnostic and therapeutic measures for TIO, however, still most clinicians are not well aware of such tumor entity due to its rarity, therefore, TIO is prone to misdiagnosis. Patients may first visit the orthopedic department, endocrinology department or have to travel to other departments which delays treatment and hence affects prognosis. Some scholars have shown that the average time from onset to correct diagnosis in TIO patients is $2.9 \pm 2.3$ years, and the average time from onset to tumor resection is $5.4 \pm 4.2$ years. The initial misdiagnosis rate of patients is $95.1 \%$ [137/144], and 144 patients have 240 misdiagnosis (18) (Table 3).

To sum up, the diagnosis strategy for TIO patients with primary tumors in the maxillofacial region is as follows: (I) an analysis of the patient's history and physical examination should be achieved to have systemic overview (whole body bone pain, muscle pain, difficult movements, with drop height) of the patients; (II) serological examination (such as low blood phosphorus, high urine phosphorus, elevated blood ALP) is mandatory; (III) imaging examination; low BMD examination confirmed phosphorus osteomalacia; (IV) the serum levels of FGF-23 in combination with the patient's family history and special history of medication, exclude inherited osteomalacia and drug induced low phosphorus osteomalacia; (V) whole body imaging scans; scintigraphy screening suspected primary tumor lesions, if still not clear; (VI) if the patient's economic condition allows, PET-CT can be added to improve the sensitivity, then surgical treatment can be performed after the tumor is found.

\section{Acknowledgments}

Funding: This study is supported by Clinical Plus Project of Shanghai 9th People's Hospital (grant number JYLJ201817 to LW), the National Natural Science Foundation of China (grant number 81771046 to Lei Wang,81970907 to LW) and Shanghai Talent Development (grant number 2018042 to $\mathrm{LW}$ ).

\section{Footnote}

Reporting Checklist: The authors have completed the CARE reporting checklist. Available at https://fomm.amegroups. com/article/view/10.21037/fomm-21-23/rc 
Peer Review File: Available at https://fomm.amegroups.com/ article/view/10.21037/fomm-21-23/prf

Conflicts of Interest: All authors have completed the ICMJE uniform disclosure form (available at https://fomm. amegroups.com/article/view/10.21037/fomm-21-23/coif). LW serves as an unpaid Managing Editor of Frontiers of Oral and Maxillofacial Medicine. LW reports that this study is supported by the Clinical Plus Project of Shanghai 9th People's Hospital (No. JYLJ201817 to LW), the National Natural Science Foundation of China (No. 81771046 to LW, No. 81970907 to LW) and Shanghai Talent Development (No. 2018042 to LW). The other authors have no conflicts of interest to declare.

Ethical Statement: The authors are accountable for all aspects of the work in ensuring that questions related to the accuracy or integrity of any part of the work are appropriately investigated and resolved. All procedures performed in studies involving human participants were in accordance with the Declaration of Helsinki (as revised in 2013). Approved by the Medical Ethics Committee of Shanghai Ninth People's Hospital, Shanghai Jiao Tong University School of Medicine (SH9H-2018-T95-2). The patient's permission for publication was obtained.

Open Access Statement: This is an Open Access article distributed in accordance with the Creative Commons Attribution-NonCommercial-NoDerivs 4.0 International License (CC BY-NC-ND 4.0), which permits the noncommercial replication and distribution of the article with the strict proviso that no changes or edits are made and the original work is properly cited (including links to both the formal publication through the relevant DOI and the license). See: https://creativecommons.org/licenses/by-nc-nd/4.0/.

\section{References}

1. McCance RA. Osteomalacia with Looser's nodes (Milkman's syndrome) due to a raised resistance to vitamin D acquired about the age of 15 years. Q J Med 1947;16:33-46.

2. Prader A, Illig R, Uehlinger E, et al. Rickets following bone tumor. Helv Paediatr Acta 1959;14:554-65.

3. Folpe AL, Fanburg-Smith JC, Billings SD, et al. Most osteomalacia-associated mesenchymal tumors are a single histopathologic entity: an analysis of 32 cases and a comprehensive review of the literature. Am J Surg Pathol
2004;28:1-30.

4. Shah R, Lila AR, Jadhav RS, et al. Tumor induced osteomalacia in head and neck region: single center experience and systematic review. Endocr Connect 2019;8:1330-53.

5. Berndt TJ, Schiavi S, Kumar R. "Phosphatonins" and the regulation of phosphorus homeostasis. Am J Physiol Renal Physiol 2005;289:F1170-82.

6. Jiang Y, Xia WB, Xing XP, et al. Tumor-induced osteomalacia: an important cause of adult-onset hypophosphatemic osteomalacia in China: report of 39 cases and review of the literature. J Bone Miner Res 2012;27:1967-75.

7. Dadoniene J, Miglinas M, Miltiniene D, et al. Tumourinduced osteomalacia: a literature review and a case report. World J Surg Oncol 2016;14:4.

8. Meng T, Zhou W, Li B, et al. Enbloc resection for treatment of tumor-induced osteomalacia:a case presentation and a systematic review. World J Surg Oncol 2015;13:176.

9. Ke YH, Zhang ZL, Zhang HZ, et al. Tumor induced osteomalacia: two case reports and literature review. Chin J Endocrinol Metab 2009;25:465-7.

10. Dorfman HD, Czerniak B. Bone tumor. St Louis: Mosby, 1998:1111-9.

11. Gardner KH, Shon W, Folpe AL, et al. Tumorinduced osteomalacia resulting from primary cutaneous phosphaturic mesenchymal tumor: a case and review of the medical literature. J Cutan Pathol 2013;40:780-4. quiz 779.

12. Zhong DR, Liu TH, Yang D, et al. Clinicopathologic study of 10 cases of osteomalacia or rickets-associated mesenchymal tumors. Zhonghua Bing Li Xue Za Zhi 2005;34:724-8.

13. Imanishi $Y$, Kobayashi K, Kawata T, et al. Regulatory mechanisms of circulating fibroblast growth factor 23 in parathyroid diseases. Ther Apher Dial 2007;11 Suppl 1:S32-7.

14. Ito N, Fukumoto S, Takeuchi Y, et al. Effect of acute changes of serum phosphate on fibroblast growth factor (FGF) 23 levels in humans. J Bone Miner Metab 2007;25:419-22.

15. Horensten ML, Boner G, Rosenfeld JB. The shrinking man. A manifestation of severe renal osteodystrophy. JAMA 1980;244:267-8.

16. Wang H, Yoshiko Y, Yamamoto R, et al. Overexpression of fibroblast growth factor 23 suppresses osteoblast differentiation and matrix mineralization in vitro. J Bone 
Miner Res 2008;23:939-48.

17. Jiang $Y$. The role of phosphorus-regulating factor in the pathogenesis of neoplastic osteomalacia[D]. Beijing: Peking Union Medical College, 2007:30-74.

18. Feng J, Jiang $\mathrm{Y}$, Wang $\mathrm{O}$, et al. The diagnostic dilemma of tumor induced osteomalacia: a retrospective analysis of 144 cases. Endocr J 2017;64:675-83.

19. Zhang S, Wang L, Wang T, et al. Value of 68 Ga-DOTATATE Positron Emission Tomography/Computed Tomography in the Localization of Culprit Tumors Causing Osteomalacia with Negative 99m Tc-HYNICTOC Single Photo Emission Computed Tomography. Zhongguo Yi Xue Ke Xue Yuan Xue Bao 2018;40:757-64.

20. Reubi JC, Waser B, Laissue JA, et al. Somatostatin and vasoactive intestinal peptide receptors in human mesenchymal tumors: in vitro identification. Cancer Res 1996;56:1922-31.

21. Hesse E, Moessinger E, Rosenthal H, et al. Oncogenic osteomalacia: exact tumor localization by co-registration of positron emission and computed tomography. J Bone Miner Res 2007;22:158-62.

22. Seufert J, Ebert K, Müller J, et al. Octreotide therapy for tumor-induced osteomalacia. N Engl J Med 2001;345:1883-8.

23. Jing $\mathrm{H}, \mathrm{Li} \mathrm{F}$, Zhuang $\mathrm{H}$, et al. Effective detection of the tumors causing osteomalacia using [Tc-99m]-HYNICoctreotide (99mTc-HYNIC-TOC) whole body scan. Eur J Radiol 2013;82:2028-34.

24. Zhang J, Zhu Z, Zhong D, et al. 68Ga DOTATATE PET/ $\mathrm{CT}$ is an Accurate Imaging Modality in the Detection of Culprit Tumors Causing Osteomalacia. Clin Nucl Med 2015;40:642-6.

25. Qari H, Hamao-Sakamoto A, Fuselier C, et al. Phosphaturic Mesenchymal Tumor: 2 New Oral Cases and Review of 53 Cases in the Head and Neck. Head Neck Pathol 2016;10:192-200.

26. Hofman MS, Kong G, Neels OC, et al. High management impact of Ga-68 DOTATATE (GaTate) PET/CT for imaging neuroendocfine and other somatostatin expressing tumours. J Med Imaging Radiat Oncol 2012;56:40-7.

27. Reubi JC, Schar JC, Waser B, et al. Affinity profiles for human somatostatin receptor subtypes SST1-SST5 of somatostatin radiotracers selected for scintigraphic and radiotherapeutic use. Eur J Nucl Med 2000;27:273-82.

28. Yamazaki Y, Okazaki R, Shibata $M$, et al. Increased circulatory level of biologically active full-length FGF-23 inpatients with hypophosphatemic rickets/osteomalacia. J
Clin Endocrinol Metab 2002;87:4957-60.

29. Mannstadt M, Lorente C, Jüppner H. Rapid detection of intact FGF-23 in tumor tissue from patients with oncogenic osteomalacia. Clin Chem 2008;54:1252-4.

30. Ryan EA, Reiss E. Oncogenous osteomalacia. Review of the world literature of 42 cases and report of two new cases. Am J Med 1984;77:501-12.

31. Evans DJ, Azzopardi JG. Distinctive tumours of bone and soft tissue causing acquired vitamin-D-resistant osteomalacia. Lancet 1972;1:353-4.

32. Weidner N, Santa Cruz D. Phosphaturic mesenchymal tumors. A polymorphous group causing osteomalacia or rickets. Cancer 1987;59:1442-54.

33. Mékinian A, Ladsous M, Balavoine AS, et al. Curative surgical treatment after inefficient long-acting somatostatin analogues therapy of a tumor-induced osteomalacia. Presse Med 2011;40:309-13.

34. Yin Z, Du J, Yu F, et al. Tumor-induced osteomalacia. Osteoporos Sarcopenia 2018;4:119-27.

35. Vahabzadeh-Hagh AM, Zipser BD, Tan HT, et al. More than just tissue diagnosis in a patient with maxillofacial bony lesions and hypercalcemia. Laryngoscope 2017;127:1318-21.

36. Jan de Beur SM, Streeten EA, Civelek AC, et al. Localisation of mesenchymal tumours by somatostatin receptor imaging. Lancet 2002;359:761-3.

37. Sharkis DH, Devereux JP, Chako AC, et al. Reversible avascular necrosis of the femoral head in tumor-induced osteoma lacia. Endocr Pract 1997;3:137-9.

38. Yavropoulou MP, Gerothanasi N, Frydas A, et al. Tumorinduced osteomalacia due to a recurrent mesenchymal tumor overexpressing several growth factor receptors. Endocrinol Diabetes Metab Case Rep 2015;2015:150025.

39. Mishra SK, Kuchay MS, Sen IB, et al. Successful Management Of Tumor-Induced Osteomalacia with Radiofrequency Ablation: A Case Series. JBMR Plus 2019;3:e10178.

40. Fukumoto S. Anti-fibroblast growth factor 23 antibody therapy. Curr Opin Nephrol Hypertens 2014;23:346-51.

doi: 10.21037/fomm-21-23

Cite this article as: Shi J, Wang L, Abdelrehem A, Zhang Y, Wu Z, Tian Z, Li S, Wang L. Tumor-induced osteomalacia caused by head and neck tumors: report of an unusual case and literature review. Front Oral Maxillofac Med 2021;3:19. 